\title{
Operator method for nonperturbative calculation of the thermodynamic values in quantum statistics: diatomic molecular gas
}

\author{
I D Feranchuk ${ }^{1}$ and A A Ivanov ${ }^{1,2}$ \\ ${ }^{1}$ Department of Theoretical Physics, Belarusian State University, 4 Fr. Skariny avenue, \\ 220080 Minsk, Belarus \\ ${ }^{2}$ Department of Natural Sciences, Belarusian National Technical University, \\ 65 Fr. Skariny avenue, 220013 Minsk, Belarus \\ E-mail: fer@open.by
}

Received 10 January 2004, in final form 2 June 2004

Published 6 October 2004

Online at stacks.iop.org/JPhysA/37/9841

doi:10.1088/0305-4470/37/42/001

\begin{abstract}
Operator method and cumulant expansion are used for nonperturbative calculation of the partition function and the free energy in quantum statistics. It is shown for Boltzmann diatomic molecular gas with some model intermolecular potentials that the zeroth-order approximation of the proposed method interpolates the thermodynamic values with rather good accuracy in the entire range of both the Hamiltonian parameters and temperature. The systematic procedure for calculation of the corrections to the zeroth-order approximation is also considered.
\end{abstract}

PACS numbers: 02.60.Gf, 03.65.Db, 05.70.Ce

\section{Introduction}

At present, most physical problems of interest can be solved neither exactly nor in terms of the canonical perturbation theory (CPT), therefore a considerable attention is given to the development of methods of nonperturbative analysis of quantum systems (see, e.g., review [1] and references therein). One such method, called the operator method (OM) of approximate solution of the Schrödinger equation, proved to be very effective. The OM was presented in [2] and approved for a number of physical problems including those with many degrees of freedom [3-8]. The main advantages of the OM are defined by the fact that its zeroth-order approximation allows one to calculate the Hamiltonian eigenvalues and eigenvectors with rather high accuracy in the entire range of both the Hamiltonian parameters and 
quantum numbers of the states. Besides, the further approximations of the OM lead to the sequences converging to the exact solutions. However, most of the particular applications of the OM have been considered before for the systems in 'pure' quantum states.

The nonperturbative methods are of great interest also in quantum statistics, when calculating thermodynamic characteristics of quantum systems. These characteristics can be expressed through either the partition function or the free energy of a system. Most of the known nonperturbative methods in quantum statistics are based on Feynman path integrals (e.g., [9] and references therein). However, an alternative representation of the partition function based on the direct summation over the energy states is also important because it could be more convenient and descriptive for some applications, especially in atomic and molecular physics when the system is characterized by a large but finite number of degrees of freedom. For example, the additional procedure of bosonization is necessary when the functional integrals are used for the spin systems, and that leads to complication of the real Hamiltonian (see, e.g., [10]).

The purpose of this paper is to generalize the OM for the nonperturbative calculation of the thermodynamic values in quantum statistics with the Schrödinger representation for quantum systems. A specific feature of this problem in comparison with application of the OM for 'pure' quantum states is that the partition function is defined by an energy spectrum as a whole and includes the summation over all states. As a result, the accuracy of calculation of the thermodynamic values for some temperature interval can be rather low even in the case when the Hamiltonian eigenvalues are found with high precision (see example in section 2). Therefore we should analyse whether the OM accuracy for the spectral problem [3] ensures the calculation of the thermodynamic values in the entire temperature range. Besides, some additional procedure should be developed for approximate summation over the states. It is shown in the paper that the regular nonperturbative method for calculation of the thermodynamic values can be developed on the basis of the OM combined with the cumulant expansion (CE) [11]. In order to approve our approach and compare it with other approximate methods, the Boltzmann diatomic molecular gas is considered and the partition function and the free energy corresponding to the molecular internal degrees of freedom are calculated. It is shown for some particular examples that the zerothorder approximation of the proposed method leads to the uniformly suitable estimation for the thermodynamic values in the entire range of both temperature and Hamiltonian parameters. The systematic procedure for calculation of the consequent approximations is also considered.

The paper is organized as follows. In section 2 we discuss some general definitions of uniformly suitable estimation that concerns any nonperturbative approach. The procedure for nonperturbative calculation of the Hamiltonian eigenvalues on the basis of the operator method is described in section 3 as the first part of the proposed approach. The approximate summation over the states by means of the cumulant expansion is considered in section 4 as the second part of our method. In order to approve this expansion, the analytical formula for interpolation of the rotational partition function of the diatomic molecular gas is obtained in the same section on the basis of the method proposed. In section 5 we use our approach for calculation of the partition function and the free energy for an equilibrium system of the anharmonic oscillators and compare the calculated values with the results of other methods. The obtained results are of interest not only as the approval of the considered method, but also for the description of thermodynamic characteristics of real molecular systems when anharmonic effects should be taken into account. 


\section{Formulation of the problem}

It is well known that if the Hamiltonian of some quantum system includes a small parameter, the regular procedure of calculation of the observable characteristics of this system can always be constructed in the form of a power series in this parameter. For example, it can be the canonical form of the perturbation theory with expansion in a power of small coupling constant $(g \ll 1)$; an expression as powers in $g^{-1}$ for the strong coupling limit; a series in terms of powers of $\hbar$ in the quasi-classical approximation; low- or high-temperature expansions for thermodynamic values. However, in most cases these expansions are the asymptotic ones, and hence cannot be used directly in a wide range of the Hamiltonian parameters. In contrast, the main goal of the nonperturbative methods is to calculate physical characteristics of a system in the entire range of its parameters. Further in the paper, when we refer to the nonperturbative calculation of some physical values, we assume that either there is no small parameter in the system, or its value lies out of the domain of applicability of the asymptotic expansions [1-3].

It is convenient to define the concept of the 'uniformly suitable estimation' (USE) in this case. Let us consider some physical variable with eigenvalues $F_{n}(\lambda)$ depending on the quantum number $n$ and the physical parameter $\lambda$ (the totality of the parameters and quantum numbers is implied for systems with many degrees of freedom). Let us introduce

Definition 1. The function $F_{n}^{(0)}(\lambda)$ is the USE for $F_{n}(\lambda)$ if the following inequality holds in the entire range of variation of the values $n$ and $\lambda$

$$
\left|\frac{F_{n}^{(0)}(\lambda)-F_{n}(\lambda)}{F_{n}(\lambda)}\right| \leqslant \xi^{(0)} .
$$

Here the parameter $\xi^{(0)}<1$ is assumed to be independent of $n$ and $\lambda$, and defines the accuracy of the USE. We also consider

Definition 2. There are the sequence of functions $F_{n}^{(s)}(\lambda)$ and the method for their calculation corresponding to the decreasing sequence of the parameters $\xi^{(s)} ; s=0,1,2, \ldots$, so that

$$
\lim _{s \rightarrow \infty} F_{n}^{(s)}(\lambda)=F_{n}(\lambda) .
$$

It seems that definition 1 is not constructive because the exact values $F_{n}(\lambda)$ are unknown in the general case. However, there are several possibilities to estimate the value $\xi^{(0)}$. In particular, one can compare the asymptotic series for $F_{n}(\lambda)$ in various limit cases either of the parameter $\lambda$ or of the quantum number $n$ with the corresponding expansions of the function $F_{n}^{(0)}(\lambda)$ taking into account that inequality (1) should hold for all cases simultaneously. Besides, an estimation for $\xi^{(0)}$ can be found as the difference between $F_{n}^{(1)}(\lambda)$ and $F_{n}^{(0)}(\lambda)$. For example, we can refer to the USE for the eigenvalues of the Hamiltonian calculated for various physical systems in 'pure' states on the basis of the OM [3-8].

A considerable advantage of the USE for various applications in comparison to the asymptotic expansions is the possibility to investigate the qualitative peculiarities of the quantum system in the intermediate range of the parameters connected with the Hamiltonian and the external conditions. At the same time, rather high precision of the USE in the zeroth order is of great use for practical calculations and it defines the rate of convergence of the consecutive approximations to the exact solution.

Let us consider from this point of view the thermodynamic perturbation theory in the Schrödinger representation of the quantum statistics. Usually, it is formulated for the free 
energy of the system [13] and the leading terms are as follows:

$$
\begin{aligned}
F(\mu, \beta)=F_{0} & +\mu \sum_{n} V_{n n} w_{n}+\mu^{2} \sum_{n} \sum_{m \neq n} \frac{\left|V_{m n}\right|^{2} w_{n}}{E_{n}^{(0)}-E_{m}^{(0)}} \\
& +\frac{1}{2} \beta \mu^{2}\left[\left(\sum_{n} V_{n n} w_{n}\right)^{2}-\sum_{n} V_{n n}^{2} w_{n}\right]+\cdots .
\end{aligned}
$$

Here we have introduced $\beta=1 / k T$ where $T$ is the temperature and $k$ is the Boltzmann constant; $F$ and $F_{0}$ are the exact and approximate free energies respectively; $E_{n}^{(0)}$ are the eigenvalues of the unperturbed Hamiltonian and $V_{m n}$ are the matrix elements of the perturbation operator with the following form of the total Hamiltonian,

$$
\hat{H}=\hat{H}_{0}+\mu \hat{V},
$$

and $w_{n}=\exp \left[\beta\left(F_{0}-E_{n}^{(0)}\right)\right]$ is the unperturbed density matrix.

Any partial sum of this series does not yield the free energy in the whole range of the temperature and the perturbation parameter $\mu$ even in the simplest cases. Let us illustrate this by means of the model Hamiltonian used earlier in [15] for the convergence analysis of a usual perturbation series for 'pure' states,

$$
\begin{aligned}
& \hat{H}=\hat{H}_{0}+\mu \hat{V}=\frac{1}{2}\left(\hat{p}^{2}+\hat{x}^{2}\right)+\mu \hat{x}^{2} \quad E_{n}^{(0)}=n+\frac{1}{2} \quad F_{0}=\frac{1}{\beta} \ln [2 \sinh (\beta / 2)] \\
& V_{m n}=\left(n+\frac{1}{2}\right) \delta_{m, n}+\frac{1}{2}\left[\sqrt{(n+1)(n+2)} \delta_{m, n+2}+\sqrt{n(n-1)} \delta_{m, n-2}\right],
\end{aligned}
$$

where $\delta_{m, n}$ is the Kronecker symbol.

Certainly, the exact free energy is well known for this model $(F=$ $\left.\frac{1}{\beta} \ln [2 \sinh (\beta / 2 \sqrt{1+2 \mu})]\right)$ but if we use these matrix elements in formula (3), then a rather simple calculation leads to the following result:

$$
F(\mu, \beta)=\frac{1}{\beta} \ln [2 \sinh (\beta / 2)]+\frac{\mu}{2} \operatorname{coth} \frac{\beta}{2}-\frac{\mu^{2}}{8}\left[1+\operatorname{coth} \frac{\beta}{2}+\frac{1}{2 \sinh ^{2} \frac{\beta}{2}}(1+2 \beta)+\cdots\right] \text {. }
$$

It is evident that this series does not satisfy the USE criteria with respect to both the essential parameters of the system. In the low temperature limit $(\beta \rightarrow \infty)$, formula (5) leads to the power series of $\mu$ for the ground-state energy and this series diverges in the range of $\mu>1 / 2$ because of a singular point of the exact eigenvalue $E_{n}=\sqrt{1+2 \mu}\left(n+\frac{1}{2}\right)$ in the complex plane of $\mu$ [15]. On the other hand, definition 1 breaks also for the free energy dependence on the parameter $\beta$. When the temperature increases $(\beta \rightarrow 0)$, the second-order correction has a stronger singularity $\left(\sim-\mu^{2} / 4 \beta^{2}\right)$ than the exact free energy.

Thus, our objective is to formulate another regular method, different from (3), which allows us to find the USE for the free energy of a quantum system with an arbitrary energy spectrum $E_{n}$.

\section{The operator method for the uniformly suitable estimation of the eigenvalues}

As mentioned above, in order to obtain the USE for the partition function one has to solve two problems: (1) to find such an approximate representation for the energy levels of a system under consideration which holds in the whole range of the Hamiltonian parameters, and (2) to perform approximate summation on quantum numbers so that the result remains uniformly 
suitable with any temperature. For the former purpose, we shall use the operator method (OM) of approximate solution of the Schrödinger equation. This method is described in detail in review paper [3]. Let us consider here only the basic expressions defining the consecutive approximations of the OM for the eigenvalues $E_{n}$ and eigenvectors $\left|\psi_{n}\right\rangle$ of a Hamiltonian $\hat{H}$ of some quantum system (the index $n$ may involve the whole set of quantum state numbers),

$$
\hat{H}\left|\psi_{n}\right\rangle=E_{n}\left|\psi_{n}\right\rangle \text {. }
$$

Let us introduce the complete set of state vectors $|n, \omega\rangle$, depending on the same quantum numbers and arbitrary parameters $\omega$. In the canonical perturbation theory (CPT) the eigenfunctions of some part $\hat{H}_{0}$ of the full Hamiltonian (zeroth-order approximation Hamiltonian) are used as such a complete set. The Schrödinger equation for that part is assumed to be exactly solvable. In terms of the OM, the vectors $|n, \omega\rangle$ are considered as a set of variational wavefunctions. Thus, the choice of these vectors is rather arbitrary and is defined by qualitative features of the system under consideration and by the possibility of rather simple calculation of the matrix elements of the total Hamiltonian $\hat{H}$. Then we can represent the solution of equation (6) in the form of expansion

$$
\left|\psi_{n}\right\rangle=|n, \omega\rangle+\sum_{k \neq n} C_{n k}|k, \omega\rangle,
$$

and choose the normalization of the exact solution as follows:

$$
\left\langle\psi_{n} \mid n, \omega\right\rangle=1 .
$$

As a result, the initial eigenvalues problem (6) exactly reduces to the system of infinite number of nonlinear algebraic equations for $E_{n}$ and coefficients $C_{n k}$ [3]:

$$
\begin{aligned}
& E_{n}=H_{n n}+\sum_{k \neq n} C_{n k} H_{n k} \quad H_{n k}(\omega)=\langle n, \omega|\hat{H}| k, \omega\rangle \\
& C_{n m}=-\left[H_{m m}-E_{n}\right]^{-1}\left[H_{m n}+\sum_{k \neq m, n} C_{n k} H_{m k}\right] \quad m \neq n .
\end{aligned}
$$

As shown in [3], the most effective method for solving this system of equations is an iterative scheme, in which the exact solutions are calculated as the limit of the sequences (and not by summation of series, as in the CPT)

$$
E_{n}=\lim _{s \rightarrow \infty} E_{n}^{(s)} \quad C_{n k}=\lim _{s \rightarrow \infty} C_{n k}^{(s)},
$$

and the consecutive approximations in (10) are calculated by means of recurrence relations

$$
\begin{aligned}
& E_{n}^{(s)}(\omega)=H_{n n}+\sum_{k \neq n} C_{n k}^{(s-1)} H_{n k} \\
& C_{n m}^{(s)}=-\left[H_{m m}-E_{n}^{(s-1)}\right]^{-1}\left[H_{m n}+\sum_{k \neq m, n} C_{n k}^{(s-1)} H_{m k}\right] \\
& E_{n}^{(0)}=E_{n}^{(1)}=H_{n n} \quad C_{n k}^{(0)}=0 .
\end{aligned}
$$

As shown for various physical systems [3-8], recurrence relations (11) rapidly converge to the exact solutions, and the choice of the parameter $\omega$ influences only the rate of this convergence. In this paper, we restrict ourselves to expressions for the energy after the second iteration (the first one coincides with the zeroth-order approximation by definition), as we are interested mainly not in strict numerical calculations, but in constructing the USE for 
the eigenvalues, the partition function and the free energy. The second iteration leads to the following approximation:

$$
E_{n}^{(2)}(\omega) \simeq E_{n}^{(0)}(\omega)+\Delta E_{n}(\omega)=H_{n n}-\sum_{m \neq n}\left[H_{m m}-H_{n n}\right]^{-1} H_{n m} H_{m n} .
$$

Strictly speaking, this expression is suitable if the diagonal matrix elements

$$
H_{k k}(\omega) \neq H_{n n}(\omega),
$$

for the considered value of the parameter $\omega$ and for all states from the set $|k, \omega\rangle$ in expansion (7).

If for some concrete values $m$ and $\omega_{m}$

$$
H_{m m}\left(\omega_{m}\right)=H_{n n}\left(\omega_{m}\right)
$$

one should extract the state $\left|m, \omega_{m}\right\rangle$ from expansion (7) and solve the secular equation in the OM zeroth-order approximation analogously to the canonical perturbation theory in the case of degenerate states [16]. Some applications of the OM for physical systems with such features were considered in $[4,5]$.

For systems with several degrees of freedom the state vector $\left|\psi_{n}, j\right\rangle$ in equation (6) may also depend on some additional index $j$. The energy level $E_{n}$ is supposed to be independent of this index. Actually, it means that the wavefunction is also the eigenvector of some operator

$$
\hat{J}\left|\psi_{n}, j\right\rangle=j\left|\psi_{n}, j\right\rangle, \quad[\hat{J}, \hat{H}]=0,
$$

which commutes with the Hamiltonian. As shown in [3], in such a case the projection operator $\hat{T}_{j}$ for the state with fixed quantum number $j$ should be used in series (7) in order to solve both equations (6) and (13) simultaneously. It leads to unessential modification of recurrence equations (11) but does not change the properties of the OM consecutive approximations.

In this paper, the generalization of the OM for quantum statistics is of main interest and thus we shall not consider the above-mentioned more complicated systems.

In spite of the formal similarity of formula (12) and the expression obtained in the second order of the CPT (see formula (3)), the former maintains some essential differences. First, the matrix elements of the operator $\hat{H}$ are calculated with the set of state vectors $|n, \omega\rangle$ for the whole Hamiltonian (and not for its unperturbed part as in the CPT). According to [3], this circumstance is essential for the convergence of the consequent approximations of the OM. In particular, this convergence depends on the numerical dimensionless parameter, defined by the ratio of the diagonal and nondiagonal matrix elements of the total Hamiltonian

$$
\xi(\omega) \simeq\left|\frac{H_{n k}}{H_{n n}}\right|_{\max } \quad k \neq n .
$$

This value is independent of the physical parameters of the Hamiltonian and defines both the convergence of the consequent approximations of the OM and the accuracy of the USE for the zeroth-order approximation of the OM [3] in accordance with definition 1.

Another specific feature of approximation (12) is that it depends on some undefined parameter $\omega$, which should be chosen so as to provide the best accuracy of the USE in the zeroth-order approximation of the OM. It was shown in [3] that the best zeroth-order approximation of the OM for the energy level with the quantum number $n$,

$$
E_{n} \simeq E_{n}^{(0)}=E_{n}^{(0)}\left(\omega_{n}\right)
$$

is achieved when $\omega=\omega_{n}$ is chosen as the solution of the equation

$$
\frac{\partial E_{n}^{(0)}(\omega)}{\partial \omega}=0
$$


It should be noted that equation (16) is not the variational principle for the excited states. The point is that the artificial parameter $\omega$ defines actually the representation for the wavefunctions, and hence, the exact eigenvalues of the Hermitian Hamiltonian should not depend on its choice. The exact condition is

$$
\frac{\partial E_{n}(\omega)}{\partial \omega}=0 .
$$

Therefore, equation (16) can be considered as the OM zeroth-order approximation for the exact condition (17).

It is essential that the optimal value of the parameter $\omega_{n}$ depends on the quantum number of the considered state. This means that the orthonormal set of basic vectors used in expansion (7) should be chosen for every state in different ways. As a result, the consecutive approximations of the OM (11) have 'local' character in the space of quantum numbers, i.e., they are calculated independently with different sets of state vectors for every state of the system. In contrast, in terms of the CPT the corrections for all quantum numbers are defined by the same spectrum and basic state vectors of the unperturbed Hamiltonian.

In this paper, we use model physical systems in order to illustrate the applicability of the $\mathrm{OM}$ in statistical physics. In particular, let us consider the quantum anharmonic oscillator (QAO) with the Hamiltonian

$$
\hat{H}=\frac{1}{2}\left(\hat{p}^{2}+\hat{x}^{2}\right)+\mu \hat{x}^{2}+\lambda \hat{x}^{4} .
$$

It is well known that the CPT expansion for this system has zero radius of convergence with respect to the parameter $\lambda$ [12]. Therefore, the QAO is widely used for approbation of various nonperturbative methods, and all approximations can be compared with the detailed numerical analysis of this problem in [12]. In the problem of the QAO the most adequate choice of the full set of state vectors for the OM algorithm is the set of eigenfunctions of the harmonic oscillator with an arbitrary frequency, playing the role of the parameter $\omega$. At the same time, it is convenient to perform all the necessary calculations of the matrix elements in an algebraic form, using the representation of the secondary quantization [2]. In such a case, the variational parameter $\omega$ can be introduced directly into the Hamiltonian by means of creation $\hat{a}^{+}$and annihilation $\hat{a}$ operators,

$$
\begin{aligned}
& \hat{x}=\frac{1}{\sqrt{2 \omega}}\left(\hat{a}+\hat{a}^{+}\right), \quad \hat{p}=-\mathrm{i} \sqrt{\frac{\omega}{2}}\left(\hat{a}-\hat{a}^{+}\right), \\
& \hat{n}=\hat{a}^{+} \hat{a} \quad\left[\hat{a}^{+} ; \hat{a}\right]=1 \quad \hat{n}|n\rangle=n|n\rangle .
\end{aligned}
$$

Some fairly simple algebraic operations with expressions (9)-(16) lead to the following results [3]:

$$
\begin{aligned}
& E_{n}^{(0)}=\frac{1}{4 \omega_{n}}\left(\omega_{n}^{2}+1+2 \mu\right)(2 n+1)+\frac{3 \lambda}{4 \omega_{n}^{2}}\left(1+2 n+2 n^{2}\right) \\
& \omega_{n}^{3}-\omega_{n}(1+2 \mu)-\frac{6 \lambda\left(2 n^{2}+2 n+1\right)}{2 n+1}=0 \\
& \Delta E_{n}^{(2)}=-\frac{(n+1)(n+2)\left[\omega_{n}\left(1+2 \mu-\omega_{n}^{2}\right)+2 \lambda(2 n+3)\right]^{2}}{16 \omega_{n}^{2}\left[\omega_{n}\left(\omega_{n}^{2}+1+2 \mu\right)+3 \lambda(3+2 n)\right]} \\
& \quad-\frac{\lambda^{2}(n+1)(n+2)(n+3)(n+4)}{32 \omega_{n}^{2}\left[\omega_{n}\left(\omega_{n}^{2}+1+2 \mu\right)+3 \lambda(5+2 n)\right]}, \\
& \Delta E_{n}^{(3)}=-\frac{1}{256 \omega_{n}^{2}}\left\{\frac{4[(n+1)(n+2)]^{3 / 2}\left[2(2 n+3) \lambda+\omega_{n}(1+2 \mu)-\omega_{n}^{3}\right]^{3}}{\left[3(2 n+3) \lambda+\omega_{n}(1+2 \mu)+\omega_{n}^{3}\right]^{2}}\right.
\end{aligned}
$$




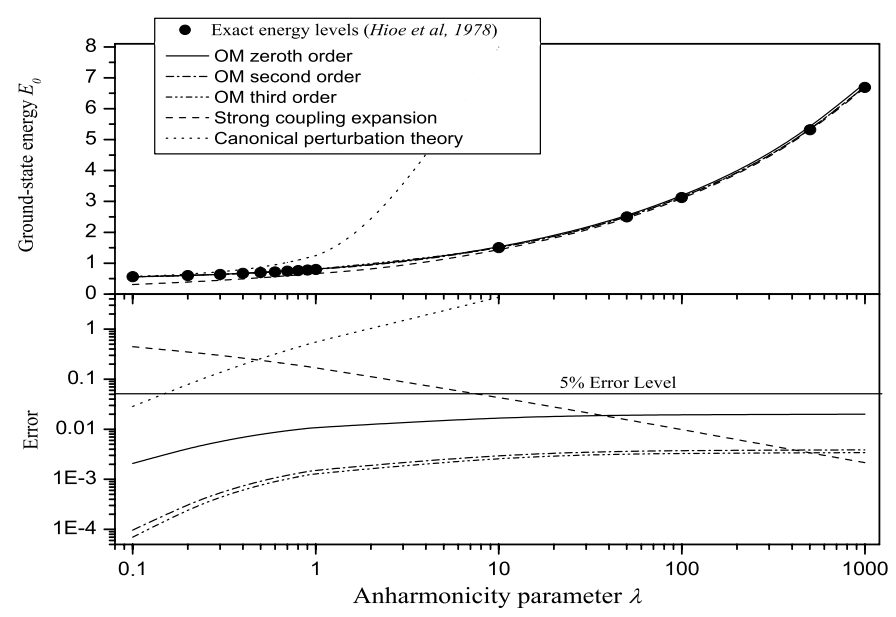

Figure 1. Various estimations for the ground-state energy of the quantum anharmonic oscillator in dependence on the anharmonicity parameter $\lambda$.

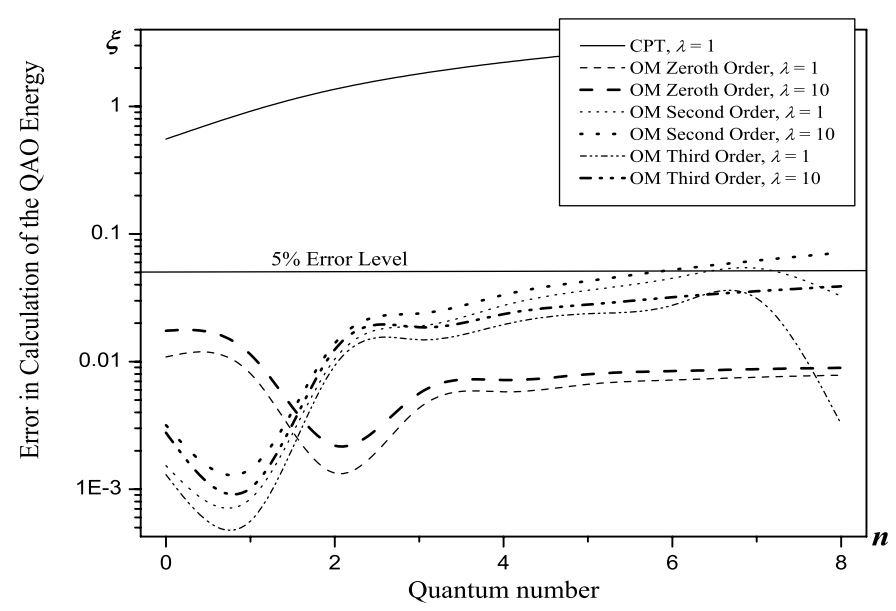

Figure 2. Errors in calculation of the energy levels of the QAO in terms of various approximations considered as the functions of quantum numbers.

$$
\begin{aligned}
& +\frac{2 \lambda[(n+1)(n+2)]^{3 / 2} \sqrt{(n+3)(n+4)}\left[2(2 n+3) \lambda+\omega_{n}(1+2 \mu)-\omega_{n}^{3}\right]^{2}}{\left[3(2 n+3) \lambda+\omega_{n}(1+2 \mu)+\omega_{n}^{3}\right]\left[3(2 n+5) \lambda+\omega_{n}(1+2 \mu)+\omega_{n}^{3}\right]} \\
& +\frac{2 \lambda^{2}(n+3)(n+4)[(n+1)(n+2)]^{3 / 2}\left[2(2 n+3) \lambda+\omega_{n}(1+2 \mu)-\omega_{n}^{3}\right]}{\left[3(2 n+3) \lambda+\omega_{n}(1+2 \mu)+\omega_{n}^{3}\right]\left[3(2 n+5) \lambda+\omega_{n}(1+2 \mu)+\omega_{n}^{3}\right]} \\
& \left.+\frac{\lambda^{3}[(n+1)(n+2)(n+3)(n+4)]^{3 / 2}}{\left[3(2 n+5) \lambda+\omega_{n}(1+2 \mu)+\omega_{n}^{3}\right]^{2}}\right\} .
\end{aligned}
$$

It can be shown by strict comparison with numerical results [12] that simple analytical expressions (20) satisfy both definitions of the USE with $\xi^{(0)} \simeq 0.03$. Figures 1 and 2 illustrate 
this statement. They show the results of calculation of the QAO energy levels in dependence on the parameter $\lambda$ and quantum number $n$, obtained by means of various methods: numerical calculations (scattered) [12]; the CPT; expansion in the strong coupling limit; the algebraic formulae (20) and (21) in the OM zeroth, second and third orders. The absolute values and relative errors of various calculation methods in dependence on the parameter $\lambda$ are compared in figures 1 and 2 shows the relative error in dependence on the quantum number $n$. One can see that only the approximation for the functions $E_{n}(\lambda)$ obtained in terms of the OM satisfies definition (1) for the USE approximated energy levels of the QAO.

\section{Operator representation for the partition function and the cumulant expansion}

Let us consider the partition function of some quantum system

$$
Z(\beta)=\sum_{n=0}^{\infty} g_{n} \exp \left[-\beta E_{n}\right]
$$

where $E_{n}$ is the energy level corresponding to a quantum number $n$ and degeneracy $g_{n}$.

To obtain the USE for this function one has to solve two problems: (1) to construct the USE for the energy spectrum of a considered system that is valid for any quantum numbers, and (2) to obtain an approximate estimation for summation on quantum numbers suitable in the whole range of temperature. It should be pointed out that for single-dimensional systems the latter problem is less important. It is sufficient to use expression (12) for the energy levels, obtained in terms of the OM, to derive the USE for thermodynamic values and to carry out direct numerical summation (see below) in (22). However, for systems with many degrees of freedom such summation is a rather complicated numerical problem, therefore we shall discuss the possibility of constructing the USE for summation on quantum states.

First, let us show that the partition function can be identically represented in the operator form as an average over some state vector. For that purpose, one can use the basic set of the state vectors considered formally as the eigenfunctions of some excitation number operator

$$
\hat{n}|n\rangle=n|n\rangle .
$$

Then one can rewrite equation (22) as

$$
Z(\beta)=\left\langle\beta^{*}\left|\exp \left[-\beta E(\hat{n})+2 \beta^{*} \hat{n}-\ln N\left(\beta^{*}\right)\right]\right| \beta^{*}\right\rangle .
$$

Here $\left|\beta^{*}\right\rangle$ is the normalized 'trial' state vector that depends on an arbitrary parameter $\beta^{*}$ having the physical meaning of an effective inverse temperature for the equilibrium system of excitations considered in (23). The actual value of $\beta^{*}$ will be defined later from the condition of the best approximation for the partition function of a real system. By definition, vector $\left|\beta^{*}\right\rangle$ is of the following form:

$$
\left|\beta^{*}\right\rangle=\sqrt{N} \sum_{n=0}^{\infty} \sqrt{g_{n}} \exp \left[-\beta^{*} n\right]|n\rangle \quad N=\left[\sum_{n=0}^{\infty} g_{n} \exp \left(-2 \beta^{*} n\right)\right]^{-1} .
$$

It is important that the state vector $\left|\beta^{*}\right\rangle$ should not be considered as some expression for the mixed state corresponding to Gibbs ensemble. Actually, equations (23)-(25) give the identical representation for the numerical (non-operator) value (22) in the form of quantum mechanical average using the auxiliary operator $\hat{n}$. It is also necessary to stress that the operational representation for the partition function, analogous to (24), can be obtained by choosing a more complicated trial vector instead of (25). Such a vector should take into account specific features of the problem under consideration. In every particular case, such a 
choice may lead to a more accurate zeroth-order approximation, but a simple vector (25) used in this paper allows us to construct the universal scheme for calculating the USE that is valid for an arbitrary quantum system.

Besides, this representation is formulated without using some specific form for the degeneracy factors $g_{n}$. In the general case, the exact calculation of this factor in advance could prove to be a rather complicated problem. But when the OM is used for the approximate calculation of the energy levels $E_{n}$ the factor $g_{n}$ can be calculated with the same accuracy by means of approximate solution of equation (13) which defines the degeneracy of the state with the quantum number $n$ [3].

Now, we can use the cumulant expansion (CE) for calculating the average value (24). Recall that the $\mathrm{CE}$ is valid for an arbitrary exponential operator when averaging over the normalized state vector [11]

$$
\langle\exp \hat{A}\rangle=\exp \left[\sum_{n=1}^{\infty} \frac{K_{n}}{n !}\right],
$$

where the cumulants $K_{n}$ are expressed in terms of the moments of the operator $\hat{A}$. This expansion as a whole is an accurate one but at the same time every cumulant corresponds to the partial summation of a usual power series. As follows from [11], the first few terms in equation (26) are given by

$K_{1}=\langle\hat{A}\rangle \quad K_{2}=\left\langle\hat{A}^{2}\right\rangle-\langle\hat{A}\rangle^{2} \quad K_{3}=\left\langle\hat{A}^{3}\right\rangle-3\langle\hat{A}\rangle\left\langle\hat{A}^{2}\right\rangle+2\langle\hat{A}\rangle^{3}$.

The general procedure of the CE can be applied to the partition function in form (24). Then successive approximations for the partition function will be calculated taking into account the corresponding cumulants. In this paper, we restrict ourselves to the first and second cumulants, which leads to the following approximation:

$$
\begin{aligned}
& Z \simeq Z\left(\beta, \beta^{*}\right)=Z_{0}\left(\beta, \beta^{*}\right) Z_{1}\left(\beta, \beta^{*}\right)=\exp \left[\langle A(\hat{n})\rangle-\ln N+\frac{1}{2}\left(\left\langle A^{2}(\hat{n})\right\rangle-\langle A(\hat{n})\rangle^{2}\right)\right] \\
& A(\hat{n})=-\beta E(\hat{n})+2 \beta^{*} \hat{n}
\end{aligned}
$$

It is obvious that with any fixed number of cumulants in formula (28) the partition function $Z\left(\beta, \beta^{*}\right)$ depends on the artificial value $\beta^{*}$, which can be considered as a variational parameter, leading to the best approximation of the CE in some order. Particularly, the zerothorder approximation $Z_{0}\left(\beta, \beta^{*}\right)$ and the first correction $Z_{1}\left(\beta, \beta^{*}\right)$ are defined by formulae

$$
\begin{aligned}
& Z_{0}=\exp \left[-\beta \bar{E}\left(\beta^{*}\right)+2 \beta^{*} \bar{n}-\ln N\left(\beta^{*}\right)\right] \\
& Z_{1}=\exp \left\{\frac{1}{2}\left[\beta^{2}\left(\overline{E^{2}}-\bar{E}^{2}\right)-4 \beta \beta^{*}(\overline{E n}-\bar{E} \bar{n})+4 \beta^{* 2}\left(\overline{n^{2}}-\bar{n}^{2}\right)\right]\right\} .
\end{aligned}
$$

Here, all values are averaged over the 'trial' distribution function corresponding to the ensemble of the excitations (23) with the degeneracy of states $g_{n}$ and the effective inverse temperature $\beta^{*}$, as for example

$$
\begin{aligned}
& \bar{E}\left(\beta^{*}\right)=N \sum_{n=0}^{\infty} g_{n} E_{n} \exp \left[-2 \beta^{*} n\right] \\
& \bar{n}\left(\beta^{*}\right)=N \sum_{n=0}^{\infty} g_{n} n \exp \left[-2 \beta^{*} n\right]=\frac{N}{2} \frac{\partial N}{\partial \beta^{*}} .
\end{aligned}
$$


Then the free energy calculated with the considered accuracy takes the following form:

$F(\beta)=-\frac{1}{\beta} \ln Z(\beta) \simeq F^{(0)}+F^{(1)}+\cdots=-\frac{1}{\beta}\left(\ln Z_{0}\left[\beta^{*}, \beta\right]+\ln Z_{1}\left[\beta^{*}, \beta\right]+\cdots\right)$.

To obtain the estimation for the partition function of the given energy spectrum, the variational parameter $\beta^{*}$ considered as the function of the real temperature $\beta$ can be defined from the condition of the best approximation in the zeroth order of the CE. As follows from (16), for this purpose one has to solve the equation

$$
\frac{\partial Z_{0}}{\partial \beta^{*}}=0 \Rightarrow \beta \frac{\partial \bar{E}}{\partial \beta^{*}}-2 \bar{n}-2 \beta^{*} \frac{\partial \bar{n}}{\partial \beta^{*}}+\frac{\partial N}{N \partial \beta^{*}}=0 .
$$

One can use the energy spectrum, obtained in terms of the OM to calculate the moments $\bar{E}, \overline{E^{2}}$ with the distribution function (30). Then the totality of expressions (12), (16), (29) and (32) leads to the USE for the thermodynamic characteristics of a system with the accuracy to the terms of second order in approximation of the OM and the CE. In some cases, the additional approximation in these equations can also be applied. This approximation leads to the worst accuracy, but permits one to simplify significantly the evaluations. It is based on the following.

Recall that condition (16) corresponds to the optimal choice of the parameter $\omega_{n}$ for the best approximation for the energy level with quantum number $n$ in the zeroth order of the OM. But convergence of the successive approximations of the OM exists for all values of $\omega$ [3]. At the same time, the partition function and the free energy are integral characteristics in the space of quantum numbers of a system. Thus, it is possible to expect that in order to construct the USE for these values it is sufficient to choose the parameter $\omega$ as the same value for all quantum numbers, and to use it for defining the best approximation condition for the free energy in the zeroth order of the OM and the CE. This means that for the calculation of the moments of the energy spectrum one has to take into account only the explicit dependence of the approximate energy spectrum on the quantum number $n$ and disregard a more complicated dependence related to the choice of $\omega_{n}$ from condition (16). Hence, the values

$$
\begin{aligned}
& \bar{E}\left(\beta^{*}, \omega\right) \simeq N \sum_{n=0}^{\infty} g_{n}\left[E_{n}^{(0)}(\omega)+\Delta E_{n}(\omega)\right] \exp \left[-2 \beta^{*} n\right] \\
& \overline{E^{2}}\left(\beta^{*}, \omega\right) \simeq N \sum_{n=0}^{\infty} g_{n}\left[E_{n}^{(0)}(\omega)\right]^{2} \exp \left[-2 \beta^{*} n\right]
\end{aligned}
$$

depend on both the parameter $\beta^{*}$ and the variational parameter $\omega$, which should be taken from the minimum condition for the free energy. As a result, instead of a system of algebraic equations (29) for $\omega_{n}$ it is necessary to solve only one equation for the parameter $\omega$

$$
\frac{\partial}{\partial \omega} \sum_{n=0}^{\infty} g_{n}\left[E_{n}^{(0)}(\omega)\right] \exp \left[-2 \beta^{*} n\right]=0 .
$$

The physical meaning of this equation is that the variational parameter $\omega$ should be chosen from the condition of the best approximation of the energy level with maximum occupancy corresponding to a given temperature.

In this paper, we illustrate the efficiency of our method in obtaining the USE for some specific systems. For these systems, all the calculations can be performed analytically. In spite of the model pattern of the problems under consideration, they are rather widely used for investigations of thermodynamic properties of real molecular gases [14], therefore, the set of analytical formulae considered below may be useful for nonperturbative analysis of anharmonic effects in such systems. 
It should be pointed out that in the case of a harmonic oscillator $(\lambda=0)$ in $(18)$, rather simple calculations show that, unlike the CPT (5), formulae (33), (34) lead to the exact expression for the partition function with any value of parameter $\mu$.

As mentioned above, the proposed method of calculating the USE for the partition function actually includes two components: the approximate summation over the quantum states and the approximate calculation of the energy spectrum. In order to illustrate how the first one works, let us obtain the USE for the partition function and the free energy for the system of quantum rotators. Such a system is used for simulation of the contribution of rotational degrees of freedom to the thermodynamic characteristics of diatomic molecular gases [13]. We can consider this system as a model with known energy spectrum and degeneracy, therefore it can be considered as a test for approbation of the cumulant expansion for approximate summation over the states with known spectrum. Hence, we should calculate the following partition function [13]

$$
Z_{r}\left(\beta, \theta_{r}\right) \equiv Z_{r}(x)=\sum_{n=0}^{\infty}(2 n+1) \exp \left[-\beta \theta_{r}\left(n^{2}+n\right)\right]
$$

Here $\theta_{r}=\hbar^{2} / 2 I$ is the rotational temperature of the system measured in energy units; $I$ is the moment of inertia of the considered molecules. Let us introduce $x$ as the dimensionless parameter, $x=\beta \theta_{r}$.

One can find analytically the asymptotic formulae for small and large temperatures which are connected with the limit cases $x \gg 1$ and $x \ll 1$ correspondingly

$$
Z_{r}(x) \simeq 1+3 \exp (-2 x)+\ldots \quad x \gg 1 .
$$

In the opposite limit case, we use two terms of the Euler formula of summation

$$
\sum_{n=1}^{\infty} f(n+a) \simeq \int_{a}^{\infty} f(y) \mathrm{d} y-\frac{1}{2} f(a)-\frac{1}{12} f^{\prime}(a),
$$

which leads to

$$
\begin{aligned}
Z_{r}(x) \simeq 1+\int_{0}^{\infty}(2 y+1) \exp \left[-x\left(y^{2}+y\right)\right] \mathrm{d} y-\frac{1}{2}-\frac{1}{12}(2-x)+\cdots \\
=\frac{1}{x}+\frac{1}{3}+\frac{x}{12} \quad x \ll 1 .
\end{aligned}
$$

The presented expressions do not describe adequately the function $Z_{r}(x)$ in the range of intermediate values of the parameter $x$. Actually $Z_{r}(x)$ can be expressed through the Weierstrass function [24], but it is rather complicated for analytical investigations. Let us show that the usage of the proposed method allows one to obtain the USE for this function in the entire range of $x$.

In such a case all average values in formula (30) can be directly expressed through moments of the 'trial' distribution function

$$
\begin{array}{ll}
N=\left[\sum_{n=0}^{\infty}(2 n+1) \mathrm{e}^{-2 \beta^{*} n}\right]^{-1}=\frac{(1-q)^{2}}{1+q} \quad q=\mathrm{e}^{-2 \beta^{*}} \\
\bar{n}=N \sum_{n=0}^{\infty} n(2 n+1) \mathrm{e}^{-2 \beta^{*} n}=\frac{q(3+q)}{1-q^{2}} & \overline{n^{2}}=\frac{q\left(3+8 q+q^{2}\right)}{(1+q)(1-q)^{2}} .
\end{array}
$$

Using the presented formulae in (29), we can obtain the following expression for the partition function in the zeroth order of the $\mathrm{CE}$ (the value $0<q<1$ can be considered as a 
variational parameter instead of $\beta^{*}$ ),

$$
\begin{aligned}
& Z_{r}^{(0)}(x, q)=\exp [\varphi(x, q)] \\
& \varphi(x, q)=-x \frac{6 q}{(1-q)^{2}}-\frac{3 q+q^{2}}{1-q^{2}} \ln q-\ln \left[\frac{(1-q)^{2}}{1+q}\right] .
\end{aligned}
$$

This formula should be supplemented with an equation for calculating the function $q=q(x)$, following from condition (32) for the best choice of the zeroth-order approximation

$$
\begin{aligned}
& \frac{\partial \varphi}{\partial q}=0 \quad x=-\frac{\left(3+2 q+3 q^{2}\right)(1-q)}{6(1+q)^{3}} \ln q=0 . \\
& Z_{r}^{(0)}(q)=\exp [\varphi(q)] \\
& \varphi(q)=q\left[\frac{3+2 q+3 q^{2}}{(1-q)(1+q)^{2}}-(3+q)\left(1-q^{2}\right)\right] \ln q-\ln \left[\frac{(1-q)^{2}}{1+q}\right] .
\end{aligned}
$$

The system of equations (40), (41) should be considered as a parametric assignment of the function $Z_{r}^{(0)}(x)$, defining the zeroth-order approximation for the partition function.

The first-order correction to the $\mathrm{CE}$ for the considered model spectrum is defined by the expression

$Z_{r}^{(1)}(x, q)=\exp \left[\varphi_{1}(x, q)\right]$

$\left.\varphi_{1}(x, q)=\frac{1}{2} x^{2}\left[\overline{\left(n^{2}+n\right)^{2}}-\overline{\left(n^{2}+n\right)}\right)^{2}\right]+2 x\left[\overline{\left[\left(n^{2}+n\right)\right.}-\overline{\left(n^{2}+n\right)} \bar{n}\right] \ln q+\left[\overline{n^{2}}-\bar{n}^{2}\right] \ln ^{2} q$.

Using equation (40) in the previous expression, one can find the following:

$$
\begin{aligned}
& Z_{r}^{(1)}(q)=\exp \left[\varphi_{1}(q)\right] \\
& \varphi_{1}(q)=\frac{q^{2}}{6} \frac{15+4 q+26 q^{2}+4 q^{3}+15 q^{4}}{(1-q)^{2}(1+q)^{6}} \ln ^{2} q .
\end{aligned}
$$

The presented formulae define the USE for the partition function of the quantum rotator. In particular, they lead to the following results in the corresponding limit cases

$$
\begin{aligned}
& x \simeq-\frac{\ln q}{2}, \quad \varphi(q) \simeq 3 q, \quad Z_{r}^{(0)}(x) \simeq 1+3 \exp (-2 x)+\cdots, \quad x \gg 1 \\
& x \simeq \frac{(1-q)^{2}}{6}, \quad \varphi(q) \simeq 1+\ln 2-2 \ln (1-q), \quad Z_{r}^{(0)}(x) \simeq \frac{0.906}{x}+0.303+\cdots, \quad x \ll 1 .
\end{aligned}
$$

Comparing these results with corresponding asymptotic formulae (36), (37), one can see that the constructed approximation describes correctly the functional dependence of the exact partition function in both limit cases. At the same time in compliance with the common definition (1) for the USE the accuracy of the estimation is defined by parameter $\xi^{(0)} \simeq 0.1$. The first-order correction (43) of the CE increases the accuracy of the estimation, in particular, when the value of $x$ is small

$$
Z_{r}(x) \simeq Z_{r}^{(0)}(x) Z_{r}^{(1)}(x) \simeq \frac{1.063}{x}+0.348+\cdots, \quad x \ll 1 .
$$

Figure 3 represents the comparison of the results of evaluations in terms of exact and approximate analytical formulae in the zeroth and second orders of the CE. It demonstrates 


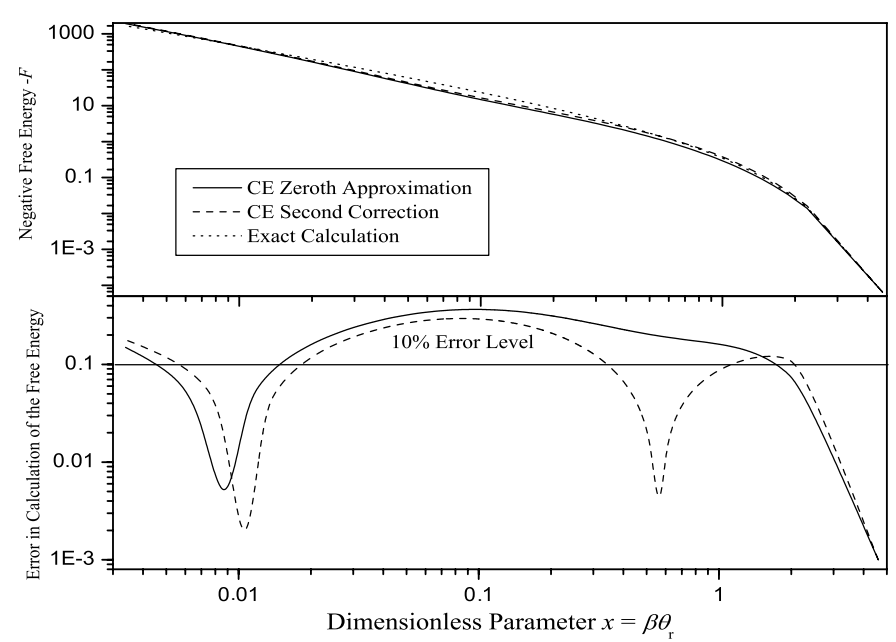

Figure 3. Free energy of quantum rotator and error in its calculation in terms of the CE in zeroth and second order in dependence on the dimensionless parameter $x$.

rather good accuracy of the zeroth-order approximation of the CE and its uniform validity for all values of the dimensionless parameter, defining the properties of the discussed system.

\section{Thermodynamics of the quantum anharmonic oscillator}

The problem considered in this section illustrates the possibilities of the discussed method in the case when the approximate calculations are used for obtaining the USE for both the eigenvalues and summation over the states. For that purpose, we use the equilibrium statistical system of quantum anharmonic oscillators (QAO). This problem has been discussed in numerous papers concerning nonperturbative approximate methods (e.g., [17-27]), but the approximation satisfying definitions (1) and (2) has not been obtained yet. As mentioned above, the solution of this problem can be useful for applications in which one should take into account the anharmonicity of the molecular oscillations (for example, when considering either the thermodynamics of gases [14] or the anharmonic effects in calculations of phonon spectra in crystals [7]).

Let us consider the QAO with parameter $\mu=0$. This can be done without loss of generality, since the parameter $\mu$ can always be excluded from the Hamiltonian by the scaling transformation of the coordinate. We thus consider

$$
\hat{H}=\frac{1}{2}\left(\hat{p}^{2}+\hat{x}^{2}\right)+\lambda \hat{x}^{4} \text {. }
$$

The USE for this system is defined by formulae (20) and (21). When obtaining the USE for the partition function and the free energy it is interesting to compare the results, which can be obtained by means of approximate calculations in two different ways: (1) direct numerical summation over the QAO states taking into account the OM energy levels and (2) evaluation of the USE in analytical form by means of the approximate summation over the states based on formulae (33), (34). To estimate the accuracy of our results we will use direct numerical calculation of the partition function $(22)\left(Z_{A}^{\text {num }}\right)$ for the QAO $\left(g_{n}=1\right)$ taking into account eight energy levels of the QAO, obtained numerically in [12]. It is essential to stress that in 

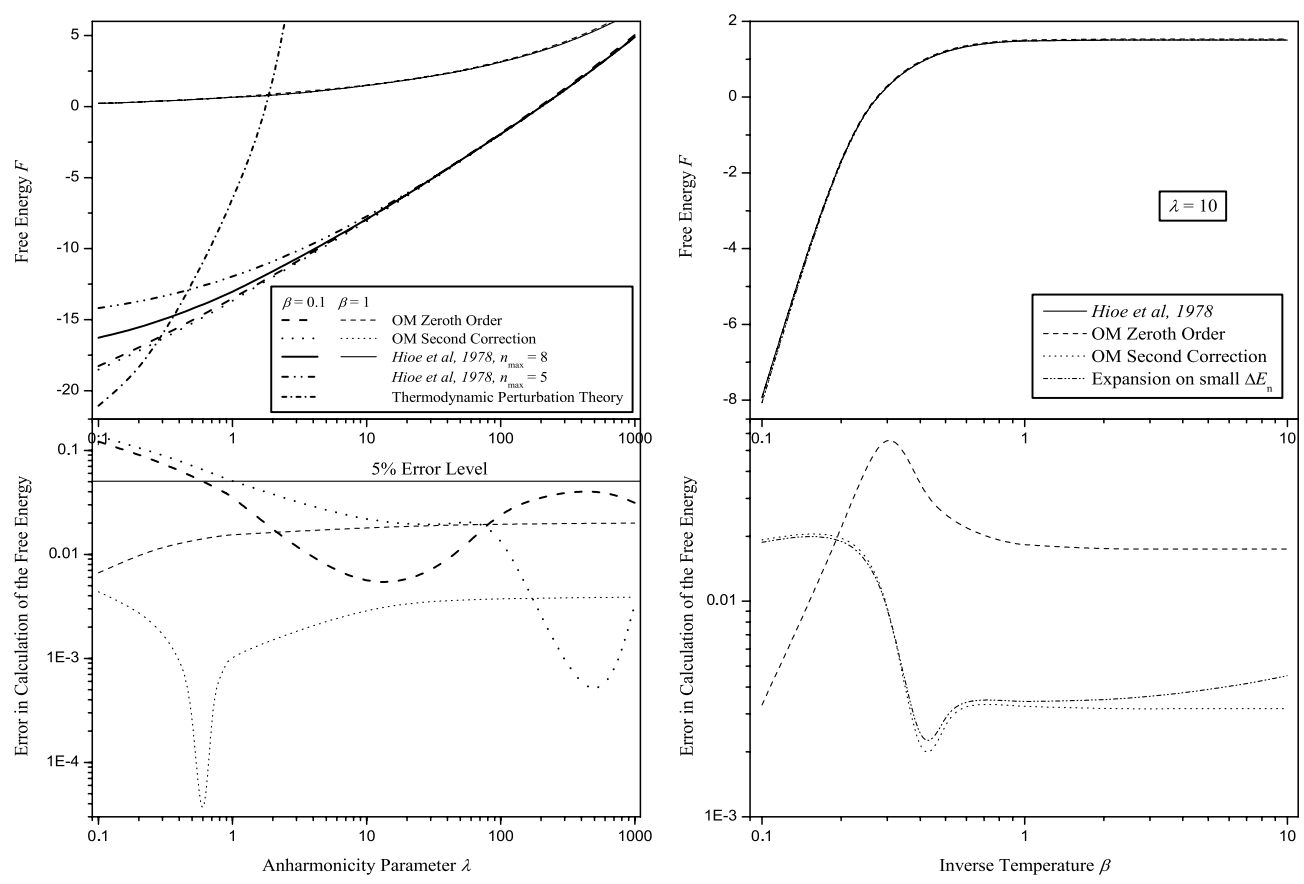

Figure 4. The free energy of the QAO using the OM zeroth and second orders and errors of these approximations in dependence on the anharmonicity parameter $\lambda$ (left panel) and on the inverse temperature $\beta$ (right panel) in comparison with the results of the numerical calculations. Lines, corresponding to $\beta=1$ in the upper part of the left panel, and all lines in the upper part of the right panel almost coincide. Results obtained in terms of the thermodynamic perturbation theory are also represented in the upper part of the left panel (they are not uniformly suitable and cannot be shown in the right panel).

real calculations with any fixed number of the QAO levels in the summation over the states, the numerical calculations lose their accuracy in the limit case of the high temperature $(\beta \rightarrow 0)$, when the whole energy spectrum becomes essential (see left panel of figure 4 ). In these cases one can use for comparison the asymptotic expressions for the partition function which can be obtained analytically:

$$
\begin{aligned}
& Z_{A}(\beta, \lambda)=\sum_{n=0}^{\infty} \exp \left[-\beta E_{n}(\lambda)\right] \simeq 1+\mathrm{e}^{-\beta E_{0}(\lambda)}, \quad \beta \gg 1 \\
& Z_{A}(\beta, \lambda) \simeq \frac{\mathrm{e}^{-\beta / 2}}{1-\mathrm{e}^{-\beta}}\left[1+\frac{3 \lambda \mathrm{e}^{-\beta}}{\left(1-\mathrm{e}^{-\beta}\right)^{2}}\right], \quad \frac{\lambda}{\left(1-\mathrm{e}^{-\beta}\right)^{2}} \ll 1 \\
& Z_{A}(\beta, \lambda) \simeq \sum_{n=0}^{\infty} \exp \left[-\beta \lambda^{1 / 3} b_{n}\right], \quad \lambda \gg 1 .
\end{aligned}
$$

Here $b_{n}$ are known numerical coefficients of the asymptotic expansion for the QAO energy levels in the strong coupling limit [12]. The analytical approximate expression for them in the OM zeroth order was obtained in [3],

$$
b_{n} \simeq\left(\frac{3}{4}\right)^{4 / 3}\left[\frac{1+2 n+2 n^{2}}{(1+2 n)^{2}}\right]^{1 / 3} .
$$


Figure 4 represents the results of comparison of the free energy $F_{A}^{\text {num }}=-(1 / \beta) \ln \left(Z_{A}^{\text {num }}\right)$ with various approximate expressions for it, which are defined as follows,

$$
\begin{aligned}
& F_{A}^{(0)}(\beta, \lambda)=-\frac{1}{\beta} \ln \left[\sum_{n=0}^{\infty} \exp \left[-\beta E_{n}^{(0)}(\lambda)\right]\right] \\
& F_{A}^{(1)}(\beta, \lambda)=-\frac{1}{\beta} \ln \left[\sum_{n=0}^{\infty} \exp \left[-\beta\left(E_{n}^{(0)}+\Delta E_{n}\right)\right]\right] \\
& F_{A}^{(01)}(\beta, \lambda)=-\frac{1}{\beta} \ln \left[\sum_{n=0}^{\infty} \exp \left[-\beta E_{n}^{(0)}\right]\left(1-\beta \Delta E_{n}\right)\right],
\end{aligned}
$$

where the energy is calculated in different orders of the OM by formulae (20), (21). We also compare our approximations with the results of the canonical thermodynamic perturbation theory [13] and the direct summation over the exact eigenvalues for the first eight levels of QAO calculated numerically in [12].

The presented results show that the zeroth-order approximation $F_{A}^{(0)}$ satisfies condition (1) and defines the USE for the pointed values with a relative error $\xi^{(0)} \simeq 0.1$. If one takes into account the subsequent iteration of the OM, the accuracy of the estimation improves. At the same time, it is necessary to stress that the expansion of the exponent in a rather small value $\Delta E_{n}$ used in expression $Z_{A}^{(01)}(\beta, \lambda)$ breaks the USE conditions in the low temperature limit $(\beta \rightarrow \infty)$. This means that the improvement in the accuracy of the zeroth-order approximation for the free energy in the subsequent iterations of the considered method has in general nonadditive character, unlike the thermodynamic CPT [13].

Let us consider now the approximate expression for the partition function obtained in terms of the zeroth-order approximation of both the OM and the CE due to formulae (32)-(34)

$$
\begin{aligned}
& Z_{A}^{(0 C)}(\beta, \lambda)=\exp \left[\varphi\left(\beta, \beta^{*}, \lambda, \omega\right)\right] \\
& \varphi\left(\beta, \beta^{*}, \lambda, \omega\right)=-\beta \overline{E_{n}^{(0)}\left(\beta^{*}, \omega, \lambda\right)}+2 \beta^{*} \bar{n}\left(\beta^{*}\right)-\ln N \\
& N=(1-q) \quad \bar{n}=\frac{q}{(1-q)} \quad \overline{n^{2}}=\frac{q+q^{2}}{(1-q)^{2}} \quad q=\mathrm{e}^{-2 \beta^{*}} \\
& \overline{E_{n}^{(0)}}=\frac{1}{4 \omega}\left(\omega^{2}+1\right)(2 \bar{n}+1)+\frac{3 \lambda}{4 \omega^{2}}\left(1+2 \bar{n}+2 \overline{n^{2}}\right)
\end{aligned}
$$

Variational parameters $q$ and $\omega$ are defined from the minimum conditions for function $\varphi$, which lead to the following equations:

$$
\begin{aligned}
& \frac{\partial \varphi}{\partial q}=0 \quad \Rightarrow \quad-\beta \frac{\omega^{2}+1}{2 \omega(1-q)}+\frac{3 \lambda(1+q)}{\omega^{2}(1-q)^{2}}+\frac{2 \ln q}{1-q}+1=0 \\
& \frac{\partial \varphi}{\partial \omega}=0 \quad \Rightarrow \quad \omega^{3}-\omega-6 \lambda \frac{1+q}{1-q}=0 .
\end{aligned}
$$

It is convenient not to solve numerically equations (51), but to consider $\lambda(q, \omega)$ and $\beta(q, \omega)$ as functions of variables $q$ and $\omega$. Together with definition (50) these functions assign in parametric form the functions $Z_{A}^{(0 C)}(\beta, \lambda)$ and $F_{A}^{(0 C)}(\beta, \lambda)=-(1 / \beta) \ln Z_{A}^{(0 C)}(\beta, \lambda)$. The results of such a calculation and its comparison with the numerically calculated free energy are shown in figure 5. As one can see, the application of the CE to summation over the states 

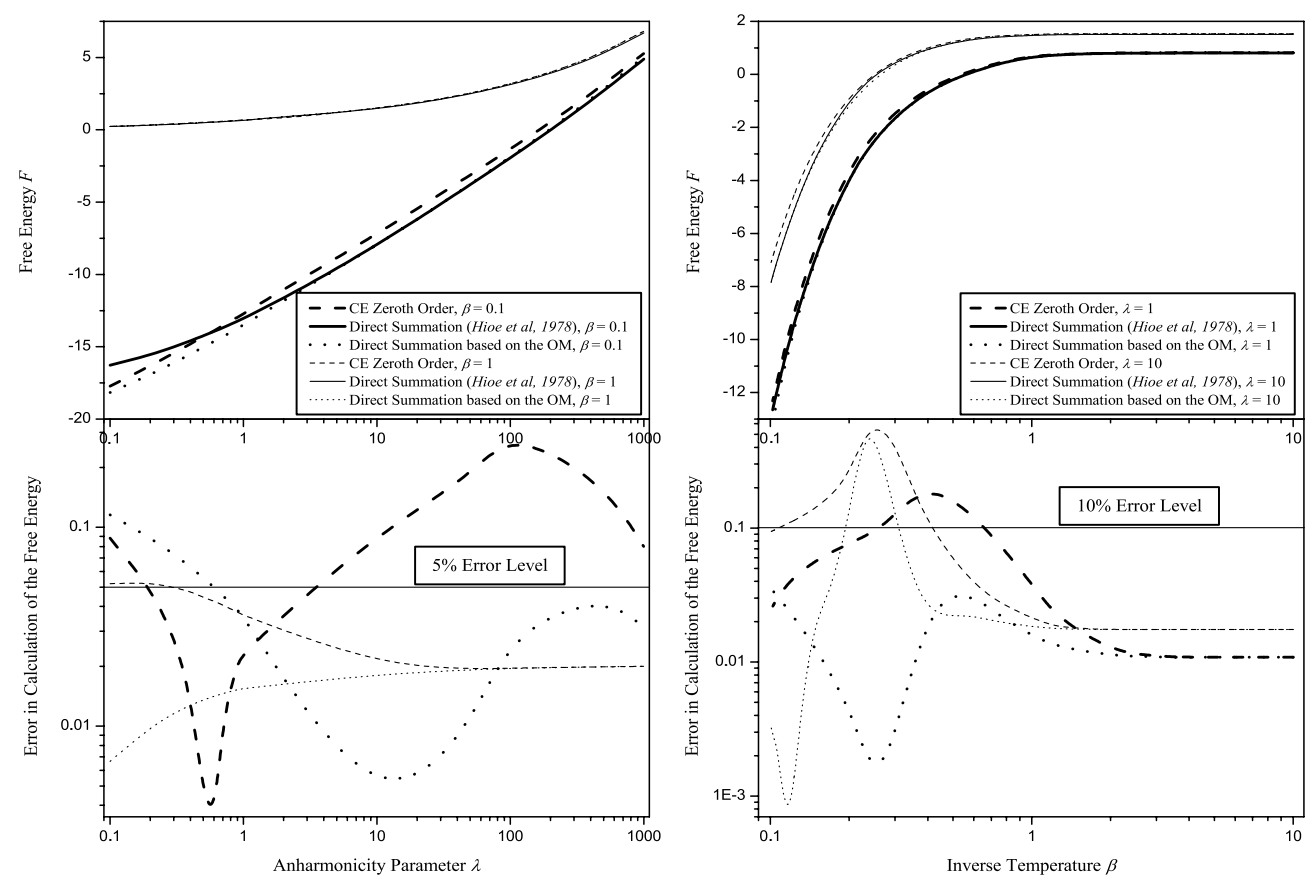

Figure 5. Approximation for the free energy of the QAO using the CE zeroth order, direct numerical summation based on the OM zeroth order and exact results [12] together with the relative errors of such approximations in dependence on the anharmonicity parameter $\lambda$ for various values of inverse temperature $\beta$ (left panel) and on the inverse temperature $\beta$ for various values of the anharmonicity parameter $\lambda$ (right panel). Lines 4,5 and 6 in the upper part of the left panel almost coincide.

keeps the zeroth-order approximation of the method under consideration satisfying the criteria of the USE with a relative error $\xi^{(0)} \simeq 0.1$. Figure 5 also shows that the values $Z_{A}^{(0 C)}(\beta, \lambda)$ and $F_{A}^{(0 C)}(\beta, \lambda)$ are rather close to the corresponding values $Z_{A}^{(0)}(\beta, \lambda)$ and $F_{A}^{(0)}(\beta, \lambda)$ calculated by means of direct summation over the OM eigenvalues $E_{n}^{(0)}\left(\omega_{n}\right)$. In the latter case the parameter $\omega_{n}$ should be defined for each level separately. The former approach based on equations (33), (34) is much easier because the parameter $\omega$ is calculated only once for each temperature. It is especially important for systems with many degrees of freedom.

To obtain the next approximation for the partition function $Z_{A}^{(1 C)}(\beta, \lambda)$ and the free energy $F_{A}^{(1 C)}(\beta, \lambda)=-(1 / \beta) \ln Z_{A}^{(1 C)}(\beta, \lambda)$ it is necessary to take into account both the correction $\Delta E_{n}$ for the energy levels obtained in terms of the OM, and the second cumulant in formula (29) for the approximate summation over the states. It is essential that in calculation of these corrections the same variational parameters $q$ and $\omega$ are used, which were found in the zeroth order. Figure 6 demonstrates the influence of these corrections on the accuracy of the USE.

It is also of interest to consider the average values of observable physical characteristics of the system (e.g., energy) in dependence on the Hamiltonian parameters and temperature. For this purpose, we use the numerical energy levels [12], the energy levels obtained in terms of the CPT and the OM in zeroth order. We consider also the expression for the average value of energy based on the cumulant expansion. Thus we obtain the following expressions for the average energy: 

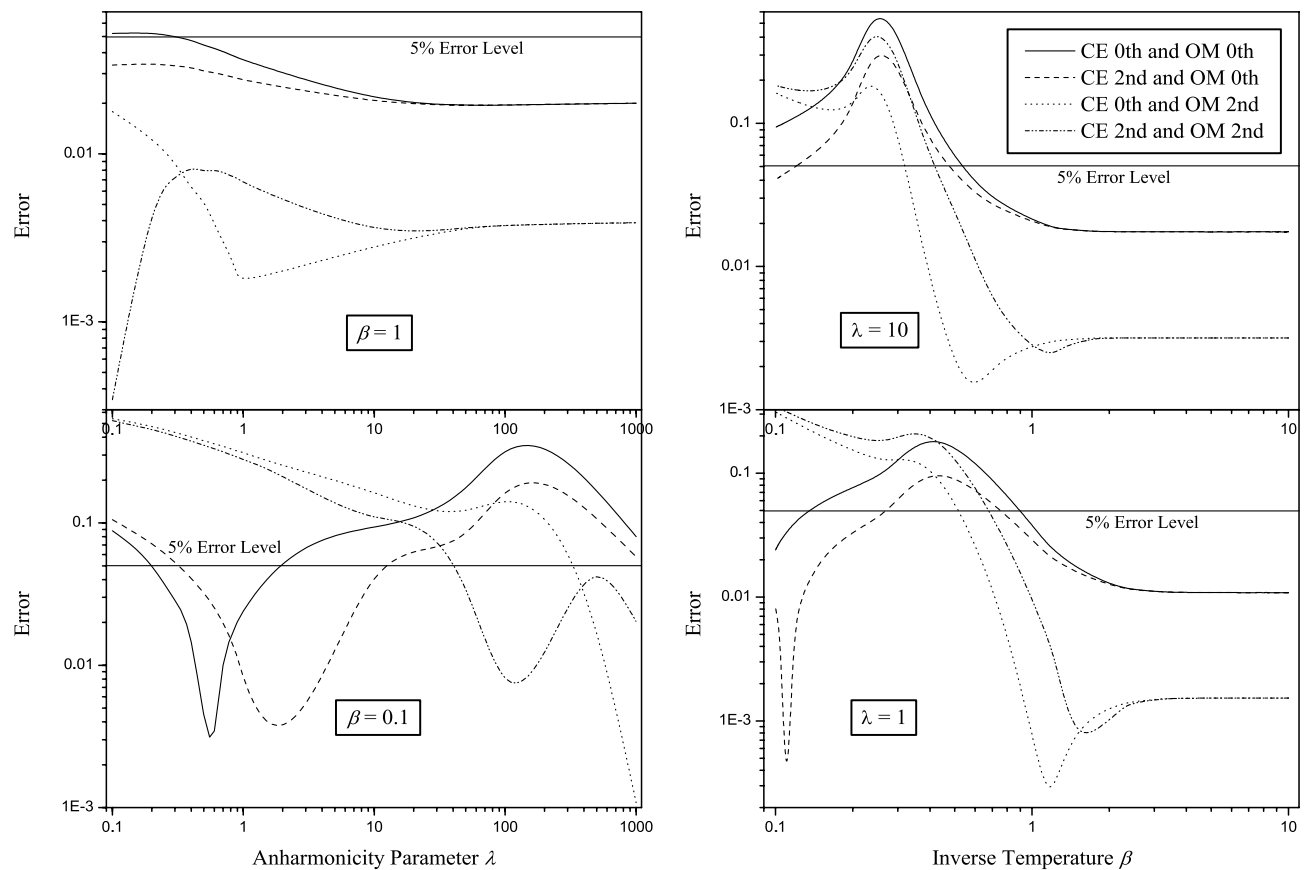

Figure 6. Errors of various approximations for the free energy of QAO in dependence on the anharmonicity parameter $\lambda$ for various values of inverse temperature $\beta$ (left panel) and on the inverse temperature $\beta$ for various values of the anharmonicity parameter $\lambda$ (right panel).
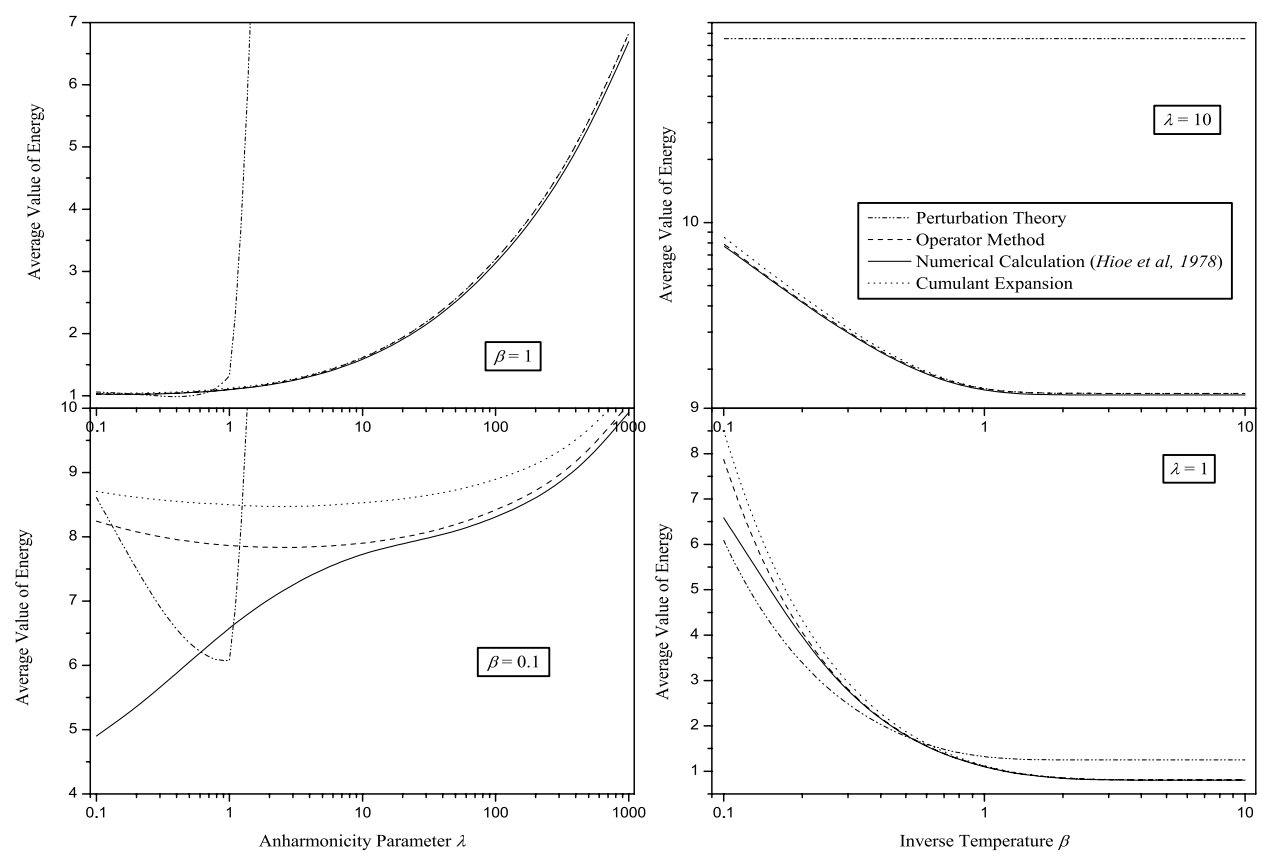

Figure 7. Average values of the energy of the QAO in dependence on the anharmonicity parameter $\lambda$ for various values of the inverse temperature $\beta$ (left panel) and on the inverse temperature $\beta$ for various values of the anharmonicity parameter $\lambda$ (right panel). 


$$
\begin{aligned}
& \overline{E^{(\text {num })}}=\frac{1}{Z_{A}^{(\text {num })}} \sum_{n} E_{n}^{(\text {num })} \exp \left[-\beta E_{n}^{(\text {num })}\right], \\
& \overline{E^{(0)}}=\frac{1}{Z_{A}^{(0)}} \sum_{n} E_{n}^{(0)} \exp \left[-\beta E_{n}^{(0)}\right], \\
& \overline{E^{(\mathrm{CPT})}}=\frac{1}{Z_{A}^{(\mathrm{CPT})}} \sum_{n} E_{n}^{(\mathrm{CPT})} \exp \left[-\beta E_{n}^{(\mathrm{CPT})}\right], \\
& \overline{E^{(C E)}}=-\frac{1}{Z^{(0 C)}} \frac{\partial}{\partial \beta} Z^{(0 C)}(\beta) .
\end{aligned}
$$

The results of these calculations are presented in figure 7 which shows that our approach leads to the uniformly suitable estimation also for the average physical values unlike the canonical perturbation theory. As was mentioned above, the noticeable deviation of the OM results from the numerical ones in the range of small $\beta$ is explained by the fact that we could use only eight eigenvalues found in [12] for calculation of $\overline{E^{(n u m)}}$. It is also important to stress again that the value $\overline{E^{(0)}}$ calculated with the OM eigenvalues depending on the set of the parameters $\omega_{n}$ is rather close to the value $\overline{E^{(C E)}}$ depending only on the parameter $\omega$ from equation (34).

\section{Conclusions}

In this paper, we have developed a nonperturbative method for calculation of the thermodynamic values of a quantum system. This has been achieved by combining the operator method of approximate solution of the Schrödinger equation and the cumulant expansion for the summation over the quantum states. The method has been approved for the Boltzmann diatomic molecular gas in order to calculate the partition function and the free energy defined by the molecular internal degrees of freedom. We have used some realistic models for the molecular movement (a quantum rotator and a quantum anharmonic oscillator) and found the uniformly suitable estimation for the thermodynamic values. This estimation tends asymptotically to the exact expansions in limit cases of both temperature and the Hamiltonian parameters. Besides, the zeroth-order approximation of the proposed method is in close agreement with the exact results (with the relative error no more than 0.1 ) in the whole range of both temperature and the Hamiltonian parameters. A systematic procedure for calculation of the subsequent corrections has been formulated and the secondorder corrections have been found to improve the accuracy of the estimation. It has also been shown that application of the cumulant expansion for the summation over the system states permits one to calculate directly the thermodynamic values without preliminary high precision estimation of the whole set of the Hamiltonian eigenvalues. It is especially important for application of the proposed algorithm for systems with many degrees of freedom.

Certainly, the strict proof of convergence of the formulated method is of special interest. Unfortunately, it seems to be a very complicated mathematical problem in the general case. But approbation of the method for a series of model systems in this paper can be considered as a qualitative argument for possibility of applying this approach to real physical problems which we suppose to analyse in the forthcoming papers.

\section{References}

[1] Yukalov V I and Yukalova E P 2002 Chaos Solitons Fractals 14839

[2] Feranchuk I D and Komarov L I 1982 Phys. Lett. A 88212 
[3] Feranchuk I D, Komarov L I, Nichipor I V and Ulyanenkov A P 1995 Ann. Phys., NY 238370

[4] Feranchuk I D, Komarov L I and Ulyanenkov A P 1996 J. Phys. A: Math. Gen. 294035

[5] Feranchuk I D and Tolstik A L 1999 J Phys. A: Math. Gen. 322115

[6] Feranchuk I D, Gurskii L I, Komarov L I, Lugovskaya O M, Burgäzy F and Ulyanenkov A P 2002 Acta Cryst. A 58370

[7] Feranchuk I D, Ulyanenkov A P and Kuzmin V S 1991 Chem. Phys. 15761

[8] Feranchuk I D and Hai L X 1989 Phys. Lett. A 137385

[9] Janke W, Pelster A, Schmidt H-J and Bachmann M (ed) 2001 Fluctuating Paths and Fields (Singapore: World Scientific)

[10] Banerjee S and Ghosh R 2003 J. Phys. A: Math. Gen. 365787

[11] Cramer H 1951 Mathematical Methods of Statistics (Princeton, NJ: Princeton University Press)

[12] Hioe F T, MacMillen D and Montroll F T 1978 Phys. Rep. 43307

[13] Landau L D and Lifsitz E M 1976 Statistical Physics (Moscow: Nauka)

[14] Gribov L A and Mushtakova S P 1999 Quantum Chemistry (Moscow: Gardariki)

[15] Fernández F M and Castro E A 1982 Phys. Lett. A 91339

[16] Landau L D and Lifsitz E M 1985 Quantum Mechanics. Nonrelativistic Theory (Moscow: Nauka)

[17] Kwek L C, Liu Y, Oh C H and Wang X-B 2000 Phys. Rev. A 62052107

[18] Vlachos K and Okopinska A 1994 Phys. Lett. A 186375

[19] Nachbagauer H 1995 Preprint ENSLAPP-A-546/95

[20] Bacus B, Meurice Y and Soemadi A 1995 J. Phys. A: Math. Gen. 28 L381

[21] Cicuta G M, Stramaglia S and Ushveridze A G 1996 Mod. Phys. Lett. A 11119

[22] Janke W and Kleinert H 1995 Phys. Rev. Lett. 752787

[23] Witschel W and Bohmann J 1980 J. Phys. A: Math. Gen. 132735

[24] Abramowitz M and Stegun A (ed) 1964 Handbook of Mathematical Functions (Washington, DC: National Bureau of Standards)

[25] Gibson W G 1984 J. Phys. A: Math. Gen. 171877

[26] Gibson W G 1984 J. Phys. A: Math. Gen. 171891

[27] Fernández F M 1991 J. Phys. A: Math. Gen. 242431

[28] Mandal S H, Ghosh R and Mukherjee D 2001 Chem. Phys. Lett. 335281

[29] Skála L, Čižek J and Zamastil J 1999 J. Phys. A: Math. Gen. 32 L123

[30] Chen G F 2001 J. Phys. A: Math. Gen. 34757

[31] Fernández F M and Pathak A 2003 J. Phys. A: Math. Gen. 365061

[32] Skála L, Čižek J and Zamastil J 1999 J. Phys. A: Math. Gen. 325715

[33] Macfarlane M H 1999 Ann. Phys., NY 271159

[34] Witschel W 2001 Physica A 300116

[35] Jafarpour M and Afshar D 2002 J. Phys. A: Math. Gen. 3587

[36] Ishikawa H 2002 J. Phys. A: Math. Gen. 354453

[37] Weissbach F, Pelster A and Hamprecht B 2002 Phys. Rev. E 66036129

[38] Mandal S 2002 Phys. Lett. A 30537

[39] Chen J-L, Kwek L C and Oh C H 2003 Phys. Rev. A 67012101

[40] Abraham K J and Vary J P 2003 Phys. Rev. C 67054313

[41] Matamala A R and Maldonado C R 2003 Phys. Lett. A 308319 